\title{
Hyalinizing trabecular adenoma of thyroid - a case report and review of literature
}

\author{
Mostafa Mahfuzul Anwar', AKM Sahabuddin Khan²
}

\begin{abstract}
:
Hyalinizing trabecular adenoma of thyroid is a rare tumour first described in 1987. It's cytological and histopathological features are very close to other thyroid neoplasms specially papillary and medullary carcinoma. So misinterpretation of cytological feature may lead to a total thyroidectomy whereas a lobectomy is enough to cure this benign condition. Here we present such a case in a 30-year-old female patient and review the diagnosis and management.
\end{abstract}

Key words: Hyalinizing trabecular adenoma; thyroid adenoma

\section{Introduction:}

Hyalinizing trabecular adenoma is a rare tumour arising in thyroid gland. It was first described as a clinicopathologic entity by Carney et al in 1987. They reported 11 patients with thyroid tumors consisting of polygonal, oval, and spindle cells arranged in a trabecular pattern and separated by a hyalinized stroma, and the term hyalinizing trabecular adenoma was introduced to describe these lesions ${ }^{1}$. Since then several cases of hyalinizing trabecular adenoma have been reported. From a practical standpoint, the most important aspect of it is that, it can mimic other thyroid neoplasms, in particular papillary and medullary carcinomas, both

1. Associate Professor, Dept of Otolaryngology and Head Neck Surgery, Chittagong Medical College, Chittagong, Bangladesh.

2. Professor, Dept of Pathology, Chittagong Medical College, Chittagong, Bangladesh.

Address of Correspondence: Dr. Mostafa Mahfuzul Anwar, MBBS, FCPS, Associate Professor, Dept of Otolaryngology - Head \& Neck Surgery, Chittagong Medical College Chittagong, Bangladesh. Email- dipro2001@yahoo.com cytologically and histologically ${ }^{2}$. Because the surgical management is commonly determined by the nature of a thyroid nodule and the result from preoperative fine-needle aspiration cytology, it is important that surgeons are aware of the features of hyalinizing trabecular adenoma. Misinterpretation of the cytological findings in a hyalinizing trabecular adenoma as suspicious or diagnostic for thyroid cancer may result in surgical "overtreatment" and total thyroidectomy may be performed when a lobectomy would have been sufficient ${ }^{2}$. The purpose of this report is to describe our recent experience with a patient diagnosed as hyalinizing trabecular adenoma and review the clinical course, diagnosis and outcome of this rare entity.

\section{Case report:}

A 30-year-old female patient presented with an oval slow growing painless swelling in right lobe of thyroid gland for the last 4 years. It was $3 \mathrm{cmX} 2 \mathrm{~cm}$ in size, nontender, firm and mobile. Regional lymph nodes were not palpable. Her CBC, blood sugar, blood urea, serum creatinine, urine R/E, X-ray chest, 
ECG, $T_{3}, T_{4}$ and TSH were within normal limit. Ultrasonography of thyroid gland showed a solid nodule in right lobe and in thyroid scan, it was a cold nodule. FNAC showed abundant follicular cells without any obvious feature of malignancy. She underwent right hemithyroidectomy in a private hospital of the city. Her post operative recovery was smooth and uneventful. The whole specimen was submitted for histopathology and diagnosed as hyalinizing trabecular adenoma.

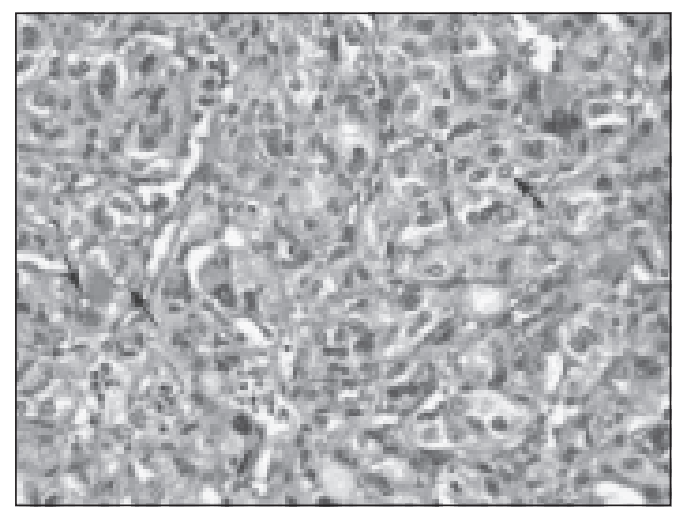

Figure 1: Histological picture of hyalinizing trabecular adenoma of thyroid.

\section{Discussion:}

Preoperative evaluation of thyroid nodules with FNAC remains the best tool to predict the type neoplasms. Diagnostic accuracy of papillary carcinoma by FNAC is quite high but unfortunately, benign thyroid lesions can not always be differentiated from malignant lesions using cytology. For example, a follicular adenoma can not be differentiated from follicular carcinoma as capsular or vascular invasion cannot be detected by this process. But strong prediction of malignancy can be made on cellular appearance and by different staining techniques ${ }^{3}$. Hyalinizing trabecular adenoma is a rare but interesting thyroid condition that can be associated with a false positive cytological evaluation, mainly because of a significant overlap of nuclear features seen in both hyalinizing trabecular adenoma and papillary cancer. Sometimes the features may mimic medullary carcinoma also. It is important for the surgeon to understand this overlap so that when this condition is encountered in pre operative evaluation, the surgeon can inform the patient of the findings and surgical plan appropriately ${ }^{2}$.

The cytology from hyalinizing trabecular adenoma is characterized by changes that are frequently seen in fine-needle aspirates from papillary carcinoma like hypercellularity, nuclear grooves, nuclear inclusions, and powdery chromatin ${ }^{4}$. Several of the cytological features of papillary cancer like nuclear grooves and hypercellularity, are nonspecific and can be seen in other conditions as well, including lymphocytic thyroiditis. lymphocytic thyroiditis has been reported to be frequently associated with hyalinizing trabecular adenoma. Other cytological changes that have been described in hyalinizing trabecular adenoma include microfollicular changes, giving rise to the suspicion of a follicular neoplasm, and amyloid-like material suspicious for medullary cancer $^{2}$.

The histological sections of the tumor show polygonal and spindle cells arranged in a trabecular growth pattern with the presence of a variable hyalinized stroma. The lesion typically occurs in middle-aged women. The absence or presence of capsular invasion can seldom be determined on frozen section. So the exact nature of the lesion typically can be determined only from permanent histopathological sections ${ }^{5}$.

As the histological changes seen in hyalinizing trabecular adenoma can be misinterpreted as medullary or papillary thyroid cancer, additional diagnostic features that differentiate the lesions are important. It 
is easier to differentiate hyalinizing trabecular adenoma from medullary carcinoma than papillary carcinoma. Both hyalinizing trabecular adenoma and medullary carcinoma are often positive for Congo red and other amyloid markers, but hyalinizing trabecular adenoma is negative for calcitonin marker ${ }^{2}$. Certain changes that are typically associated with papillary cancer, such as nuclear grooves and occasional psammoma body-like formations, can also be seen in hyalinizing trabecular adenoma. Changes that have been reported to be more prevalent in hyalinizing trabecular adenoma than in papillary carcinoma can be helpful in establishing the diagnosis. These include pronounced accumulation of basement membrane material giving rise to characteristic stromal deposits seen in FNAC, cytoplasmic yellow bodies and immunoreactivity against the MIB1 antibody $6,7,8$.

The concept of hyalinizing trabecular adenoma being a separate entity has been challenged and it has been suggested that hyalinizing trabecular adenoma may represent a variant of papillary carcinoma. This was based on nuclear changes similar to those seen in papillary carcinoma, as well as the finding that several hyalinizing trabecular adenomas expressed the papillary cancer-specific proto-oncogene RET, both at the gene and protein levels. In addition, papillary cancer and hyalinizing trabecular adenoma were reported to have similar patterns of certain cytokeratins $\mathrm{s}^{9,10}$. However, in a recent and detailed analysis of the expression of different cytokeratins in hyalinizing trabecular adenomas and papillary cancers, evidence was found that the 2 lesions express distinct and different cytokeratin patterns. These results suggest that hyalinizing trabecular adenoma is indeed a separate entity different from papillary cancer $^{2}$. This was further supported by a selective membrane and cytoplasmic immunoreactivity for MIB-1 in hyalinizing trabecular adenoma, which is completely absent in papillary thyroid carcinoma ${ }^{8}$. In addition, hyalinizing trabecular adenomas are mostly negative or only weakly positive for galectin-3, whereas most papillary thyroid cancers show strongly positive staining for this marker ${ }^{11}$. Molecular profiling may ultimately prove to be the most powerful method to differentiate hyalinizing trabecular adenoma from other thyroid neoplasms ${ }^{12}$.

\section{References:}

1. Akin MRM, Nguyen GK. Fine needle aspiration biopsy cytology of hyalinizing trabecular adenomas of the thyroid. Diagn Cytopathol 1999; 20(2): 90-4.

2. Evenson A, Mowschenson $\mathrm{P}$, Wang $\mathrm{H}$, Connolly J, Mendrinos S, Parangi S, Hasselgren PO. Hyalinizing trabecular adenoma- an uncommon thyroid tumor frequently misdiagnosed as papillary or medullary thyroid carcinoma. Am J Surg 2007; 193(6): 707-12.

3. Volante M, Papotti M. a practical diagnostic approach to solid/trabecular nodules in the thyroid. Endocr Pathol 2008; 19(2): 75-81.

4. Goellner JR, Carney JA. Cytologic features of fine needle aspirates of hyalinizing trabecular adenoma of the thyroid. Am J Clin Pathol 1989; 91(2): 115-9.

5. McCluggage WG, Sloan JM. Hyalinizing trabecular carcinoma of thyroid. Histopathology 1996; 28(4): 357-62.

6. Kuma S, Hirokawa M, Miyauchi A, Kakudo K, Katayama S. Cytologic features of hyalinizing trabecular adenoma of the thyroid. Acta Cytol 2003; 47(3): 399-404. 
7. Casey MB, Sebo TJ, Carney JA. Hyalinizing trabecular adenoma of the thyroid gland: cytologic features in 29 cases. Am J Surg Pathol 2004; 28(7): 859-67.

8. Casey MB, Sebo TJ, Carney JA. Hyalinizing trabecular adenoma of the thyroid gland identification through MIB1 staining of fine needle aspiration biopsy smear. Am J Clin Pathol 2004; 122(4): 506-10.

9. Cheung CC, Boerner SL, McMillan CM, Ramyar L, Asa SL. Hyalinizing trabecular tumor of the thyroid: a variant of papillary carcinoma proved by molecular genetics. Am J Surg Pathol 2000; 24(12): 1622-6.

10. Papotti M, Volante M, Giuliano A. RET/ PTC activation in hyalinizing trabecular tumors of the thyroid. Am J Surg Pathol 2000; 24(12): 1615-21.

11. Gaffney RL, Carney JA, Sebo TJ. Galectin-3 expression in hyalinizing trabecular tumors of the thyroid gland. Am J Surg Pathol 2003; 27(4): 494-8.

12. Finley DJ, Lubitz CC, Wei C, Zhu B, Fahey TJ. Advancing the molecular diagnosis of thyroid nodules: defining benign lesions by molecular profiling. Thyroid 2005; 15(6): 562-8. 